\title{
Anna Volodina
}

\section{A4.4 Ebenen der Verknüpfung}

Wenn eine spezifische Konnektorkonstruktion zwischen den von den Konnekten ausgedrückten Propositionen keinen Sinn ergibt, die Konstruktion als Ganze aber als wohlgeformt empfunden wird, so verknüpft der Konnektor epistemisch bewertete Propositionen oder er operiert auf der Ebene der Sprechakte. Ein Beispiel dafür ist (81), das bereits seit Austin (1961) in der Linguistik viel diskutiert wird.

(81) Es gibt Plätzchen auf dem Sideboard, wenn du welche willst.

Eine Konditionalrelation der Art, bei der sich das Antezedens auf die Proposition des Konsequens bezieht, wie z.B. in (81a), kann in diesem Fall nicht gemeint sein. Sinnvoll ist dagegen eine Interpretation wie in (81b):

(81a) Für den Fall, dass du die Plätzchen willst, sind die Plätzchen auf dem Sideboard.

(81b) Für den Fall, dass du Plätzchen willst, teile ich dir mit, dass sie auf dem Sideboard sind.

An Beispiel (81c) wird darüber hinaus deutlich, dass die Interpretation auch durch formale Merkmale gesteuert werden kann: Das interne Konnekt ist in die Struktur des externen Konnekts weder syntaktisch eingebettet noch prosodisch integriert, eine propositionale Interpretation der konditionalen Relation ist hier nicht möglich.

(81c) Wenn du Plätzchen willst, sie sind auf dem Sideboard.

D.h., während für (81) zumindest ein Märchenszenario denkbar wäre, in dem die Existenz von Plätzchen auf dem Sideboard tatsächlich von dem Willen des Zuhörers abhängt (vgl. 81a), ist für (81c) eine solche Interpretation gar nicht möglich. Wie solche Unterschiede systematisch analysiert werden können, wird im Folgenden an Beispielen erläutert. 


\subsubsection{Eine Relation - mehrere Lesarten: Das Konzept des Drei-Ebenen-Modells}

Die genannten Unterschiede in der Interpretation und damit korrelierende Formeigenschaften werden in der Linguistik etwa seit Anfang der 90 er-Jahre ${ }^{27}$ besonders intensiv diskutiert. Sprachübergreifend liegen gut dokumentierte Untersuchungen insbesondere zu den Kausalen und Konditionalen vor, auch unter Berücksichtigung gesprochensprachlicher Daten: z.B. zum Deutschen Keller (1993, 1995), Günthner (1993), Pasch (1997), Scheutz (1998a, b, 2001), Pittner (1999), Breindl (2009), Breindl/ Walter (2009), Antomo/Steinbach (2010), Volodina (2011a, b), Reis (2013); außerdem Athanasiadou/Dirven (2000) zum Englischen, Pander Maat/Sanders (2000) und Pander Maat/Degand (2001) zum Niederländischen, Degand/Pander Maat (2003) zum Französischen und Niederländischen, Pit (2003) zum Niederländischen, Deutschen und Englischen, Kitis (2006) zum Griechischen, Huelva Unternbäumen (2005) zum Spanischen, Dancygier (2009) zum Polnischen, Niederländischen und Englischen sowie ein Großteil der Beiträge des Sammelbands Causal Categories in Discourse and Cognition von Sanders/Sweetser (Hg.) (2009) zum Niederländischen und Englischen. Alle oben genannten Arbeiten nehmen in unterschiedlichem Maße Bezug auf die kognitive Theorie von Eve Sweetser (1990), die anders als ihre Vorgänger nicht von zwei Bezugsgrößen - propositional (81a) vs. nicht-propositional (81b/c) - ausgeht, sondern mit der Annahme einer im Sinne von Lakoff/Johnson (1980) metaphorischen $^{28}$ Extension von drei „Gebrauchsdomänen“ spricht: Nach Sweetser kann eine Äußerung auf drei kognitiven Bezugsebenen interpretiert werden - der propositionalen Ebene (content domain), der epistemischen Ebene (epistemic domain) und der Sprechaktebene (speech act domain).

Diese unterscheiden sich voneinander wie folgt:

a) Auf der propositionalen Ebene besteht eine Relation zwischen zwei Propositionen, die auf Sachverhalte in einer realen oder möglichen Welt referieren;

b) auf der epistemischen Ebene wird eine Proposition in Relation zu einer epistemisch bewerteten Proposition gesetzt;

c) und schließlich wird auf der Sprechaktebene eine Proposition in Relation zu einem Sprechakt gesetzt.

27 Auch wenn die Unterscheidung nach unterschiedlichen Bedeutungsebenen von Konnektoren bereits in den Arbeiten von Halliday/Hasan (1976), Pasch (1982) und Granito (1984) zumindest ansatzweise gemacht wurde, setzte sich doch erst das Modell von Sweetser (1990) in der linguistischen Forschung durch.

28 Der in Sweetser verwendete Begriff Metapher oder konzeptuelle Metapher geht im Wesentlichen auf Lakoff/Johnson (1980) zurück. Für sie ist Metapher eine wesentliche Komponente unseres Denkens. Mit der Metaphorisierung erklärt die kognitive Linguistik den assoziativen Sprung, der durch Analogiebildung zwischen zwei sprachlich ähnlichen, distinktiven konzeptuellen Bereichen gemacht wird. 
Die propositionale Ebene gilt dabei als die Basisebene, bei den beiden anderen handelt es sich in der Terminologie von Sweetser um metaphorische Ebenen. Anders als propositionale Verknüpfungen sind Verknüpfungen auf der epistemischen Ebene und auf der Sprechaktebene dahingehend asymmetrisch, dass die Relata von unterschiedlichem Typ sind und immer nur eines der Relata metaphorisch verwendet wird. Diese Beobachtung ist insbesondere für die konditional basierten Relationen wichtig, weil es immer die Proposition des Konsequens einer konditional basierten Relation ist, die metaphorisch verwendet wird. Das Antezedens wird dagegen stets propositional interpretiert. Ob in einem gegebenen Fall eine Relation auf einer der metaphorischen, „abstrakten“ Ebenen vorliegt oder auf der propositionalen, „konkreten“ Ebene, ist letztendlich darauf zurückzuführen, ob eine spezifische Relation zwischen den propositionalen Gehalten der Konnekte interpretierbar ist oder nicht. Dies beeinflusst aber nicht die Bedeutung von Konnektoren als Verknüpfer dieser Relationen. Denn Sweetser postuliert, dass alle Konnektoren unabhängig von der Art der Verknüpfung eine einzige Grundbedeutung haben. Die Möglichkeit, auf mehreren Bezugsebenen zu verknüpfen, konstituiert keine lexikalische Mehrdeutigkeit eines Konnektors. Da die gewünschte Interpretation sich im Falle der propositionalen Lesart aus dem Kontext bzw. dem allgemeinen Weltwissen und im Fall der epistemischen und sprechaktbezogenen Lesart aus dem gemeinsamen Wissen über die Situation ergibt, plädiert Sweetser für diejenigen Konnektoren, die auf allen Bezugsebenen verknüpfen können, für die Annahme der sogenannten „pragmatic ambiguity“ anstatt von lexikalischer Polysemie. Bezogen auf das in diesem Handbuch zugrunde gelegte Verständnis der Bedeutungen von Konnektoren könnte man auch sagen: Die Bedeutung des Konnektors bleibt gleich, weil die Relatrollen als kausale Rollen GRUnD und FoLGE konstant bleiben. Lexikalische Polysemie eines Konnektors liegt nur dann vor, wenn mit dem Wechsel der Ebene auch ein Wechsel in eine andere semantische Klasse verbunden ist und damit die Relatrollen verschieden sind (s. auch A3). So ist der Adverbkonnektor schließlich bei propositionaler Verknüpfung temporal (im Sinne von „Abschluss einer Sequenz“) und bei nicht-propositionaler Verknüpfung kausal (im Sinne von „denn“) zu lesen:

(82) Sie lasen weiter, bissen sich an neuen Sätzen fest, um schließlich doch zu verstehen. (die tageszeitung, 24.12.1986, S. 5) PRO $_{\text {TEMP }}$ Ich will der Telefonseelsorge nicht in ihre Arbeit reden. Sie hat schließlich mehr Erfahrung als ich.

EPS $_{\text {KAUS }}$

(Beispiele aus Breindl/Waßner 2006: 53)

Für das HDK-2 ist der Ansatz von Sweetser insofern interessant, als damit die Existenz gewisser Korrelationen zwischen einem Bündel formaler Mittel wie syntaktischer und prosodischer Struktur, syntaktischem und lexikalischem Typ des Konnektors und der Interpretation der Relation auf einer der Ebenen voraussagbar ist. Mit ihrem Ansatz lässt sich zum Beispiel der Unterschied zwischen den weil-V2- und weil-VL-Strukturen 
synchron durch unterschiedliche Art der Ebenenverknüpfung erklären (vgl. Keller 1993). Die Unterscheidung nach drei verschiedenen Arten der Verknüpfung hat sich zumindest im kausalen Bereich - mittlerweile in Grammatiken sowie einschlägigen Beiträgen zu Handbüchern (GDS, Duden-Grammatik 2005, Fabricius-Hansen 2007) durchgesetzt. Vielfach wird die These vertreten, dass es in vielen Sprachen Konnektoren gibt, die hinsichtlich der Bezugsebene vollspezifiziert sind, und solche, die unterspezifiziert sind (vgl. Sweetser 1990, Keller 1993, Crevels 2000, Sanders/Sweetser (Hg.) 2009). In HDK-1 wird in Anlehnung an Sweetser zwischen Konnektoren unterschieden, die auf allen drei Ebenen verknüpfen können - wie weil, wenn, obwohl und solchen, die aufgrund ihrer syntaktischen Einschränkung und informationsstruktureller Eigenschaften eingeschränkter verwendet werden und nur auf der epistemischen Ebene und auf der Sprechaktebene verknüpfen wie z.B. denn, da, nämlich und zumal (vgl. kritisch Breindl/Walter 2009); vollspezifiziert schließlich sei indem, das ausschließlich propositionale Verknüpfungen zulässt.

Der Ansatz Sweetsers ist in manchen Punkten problematisch. Der vielleicht wichtigste ist, dass ihr Ansatz vorsieht, dass die Verknüpfungen einer Ebene über die Relationen hinweg eine Klasse mit gemeinsamen Eigenschaften bilden können. Wenn dies so wäre, würden die formalen Merkmale mit den Ebenen relationsübergreifend und unabhängig von der syntaktischen Subklasse des Konnektors korrelieren, was nicht der Fall ist (vgl. Volodina 2011a). Ein weiterer wesentlicher Nachteil ist die nicht ausreichende Differenzierung zwischen den Bezugsebenen, die von Sweetser anhand konstruierter Einzelbeispiele diskutiert wird. Dies führt dazu, dass von den anderen, mit derselben Problematik beschäftigten Autoren sowohl feinkörnigere Unterscheidungen in Erwägung gezogen werden, in denen mehrere Arten von Sprechaktlesarten bzw. epistemischen Lesarten unterschieden und weitere Bezugsebenen eingeführt werden (vgl. etwa das Modell der ,layered structure“ der funktionalen Grammatik bei Dik et al. 1990, Crevels 2000, ferner Lang 2000), als auch gröbere Unterscheidungen wie etwa die Unterscheidung nur nach propositionalen vs. nicht-propositionalen Lesarten (Motsch/Pasch 1987) bzw. semantischen vs. pragmatischen Relationen (Knott/ Sanders 1998). Wir gehen im Folgenden, wie in der GDS und auch schon in HDK-1 praktiziert, von einer Dreiergliederung aus, wobei wir uns im Klaren sind, dass wir in vielen Fällen zugunsten einer exemplarischen Vorgehensweise von den Generalisierungen über alle Relationen und syntaktischen Verknüpfungsverfahren hinweg absehen und diesbezüglich Einschränkungen vornehmen müssen, damit die Bedeutungsund Formunterschiede zwischen Bezugsebenen adäquat dargestellt werden können. Die Unterschiede zwischen den Bezugsebenen werden im Weiteren in A4.4.2 aus funktionaler und in A4.4.3 aus formaler Sicht erläutert. 


\subsubsection{Funktionale Unterschiede zwischen den Ebenen}

Für den weiteren Umgang mit den Ebenen müssen diese in Bezug auf Konnektoren definiert werden. Eine solche Definition kann nur funktional sein, die Korrelation zu formalen Merkmalen kann dann erst in einem zweiten Schritt aufgezeigt werden. Für die Darstellung der funktionalen Unterschiede zwischen den Ebenen wird hier ein allgemeines formales Schema verwendet, das die jeweilige mögliche metaphorische Verwendung des KonSEQuens berücksichtigt.

\subsubsection{Die propositionale Ebene}

Auf der propositionalen Ebene besteht eine Relation zwischen zwei Propositionen, die auf Sachverhalte in einer realen oder möglichen Welt referieren, nämlich bei konditional basierten Subjunktorrelationen zwischen dem propositionalen Gehalt des Antezedens (Antez) und dem des Konsequens (Konseq)

$$
\begin{aligned}
& \text { Wenn }[\text { Peter den Zug verpasst }]_{\text {ANTEZ }},[\text { kommt er zurück }]_{\text {KonSEQ }} \\
& \text { KOND(AnteZ, Konseq) } \\
& \left.[\text { Peter ist zurückgekehrt }]_{\text {KoNSEQ }} \text {, weil [er Maria liebt }\right]_{\text {ANTEZ }} \\
& \text { KAUS(Antez, Konseq) } \\
& \left.[\text { Peter liebt Maria] }]_{\mathrm{ANTEZ}} \text {, weshalb [er zurückgehrt ist] }\right]_{\text {KonSEQ }} \\
& \text { KAUS(Antez, Konseq) }
\end{aligned}
$$

Prinzipiell kann das Konsequens durch das externe, aber auch durch das interne Konnekt ausgedrückt werden: Anders als die Subjunktoren wenn in (84) und weil in (85) ist der Postponierer weshalb in (86) ein KonSEquens-Marker, das Verhältnis zwischen den Relatrollen bleibt aber erhalten, so dass (85) und (86) mindestens eine gemeinsame Lesart haben.

Wenn man das relationale Verhältnis zwischen den propositionalen Strukturen mit $\mathbf{R}$ bezeichnet, so kann die Basisbedeutung für alle konditional basierten Subjunktorrelationen auf der propositionalen Ebene als R(Antez, KonseQ) festgelegt werden.

\subsubsection{Die epistemische Ebene}

Die epistemische Ebene kann laut Sweetser (1990) durch folgende Oppositionen zur propositionalen Ebene erfasst werden: 
I. fact in the world vs. mental state

II. merely describing vs. reasoning

III. objective statement vs. subjective statement

Während also im Falle der propositionalen Ebene das Konsequens auf einen Sachverhalt in einer realen oder möglichen Welt referiert, bezeichnet das KonSEQuENS bei epistemischen Relationen in der Regel mentale Zustände, d.h. Annahmen, Wünsche, Befürchtungen etc. Darüber hinaus kommen epistemische Relationen in der Regel in Kontexten der Beweisführung oder in Begründungszusammenhängen vor. Schlussendlich interagieren epistemische Relationen häufiger mit dem Faktor Subjektivität (vgl. dazu z.B. Nuyts 2001, Pit 2003, Stukker 2005), was dann der Fall ist, wenn der Sprecher sich als einzige Quelle für die Evidenz seiner Aussage ausweist und gleichzeitig seine persönliche Einstellung zur Qualität der Evidenz in Form von Bewertungen, Meinungen oder Gefühlen markiert (Frohning 2007: 71).

Ebenfalls anders als auf der propositionalen Ebene besteht die Relation auf der epistemischen Ebene nicht unmittelbar zwischen den durch die Konnekte ausgedrückten Propositionen, sondern das Konsequens ist die Annahme oder Einstellung des Sprechers zum propositionalen Gehalt des externen Konnekts. Diesen Kontrast illustriert Sweetser an einem Beispielpaar. Das propositional zu lesende (87) wird mit dem Beispiel (88) kontrastiert, dessen Lesart nur auf einer der nicht-propositionalen Ebenen zugänglich ist.

$$
\begin{array}{ll}
\text { John came back because he loved Mary. } & \text { PRO } \\
\text { John loved Mary, because he came back. } & \text { EPS }
\end{array}
$$

(Beispiele leicht verändert nach Sweetser 1990: 77)

Der zentrale Unterschied zwischen (87) und (88) besteht für Sweetser darin, dass in (87) die Tatsache, dass John Mary liebt, die Ursache dafür ist, dass John zurückgekommen ist. Dagegen ist in (88) die Tatsache, dass John zurückgekommen ist, nicht als Ursache dafür anzusehen, dass er Mary liebt. Vielmehr wird hier aus der Tatsache, dass John zurückgekommen ist, darauf geschlussfolgert, dass er Mary liebt.

Aus diesem Grund wird in (88a) ein analoges Schema wie für die Relationen der propositionalen Ebene vorgeschlagen, mit dem Unterschied, dass in (88) das KoNSEQUENS die Ursache nicht der Liebe, sondern der Annahme der Liebe Johns Mary gegenüber ist:
KAUS (ANTEz, Konseq)
PRO
(88a) KAUS (AnTEz, ANNAHME (KonsEQ))
EPS

Zur Erstellung eines allgemeinen Schemas für epistemische Relationen reicht das in (88a) angegebene Muster noch nicht aus, da die Annahme des KonsEQuens nur einen Sonderfall darstellt. Andere Typen von metaphorischen Modifikationen wie Hoff- 
nung, Bedauern, Wunsch, Befürchtung etc. gehören ebenfalls in diese Kategorie. Deswegen wird vorgeschlagen, einen allgemeineren Operator einzusetzen, der alle diese Modifikationen abdeckt, und zwar EPS (epistemische Einstellung bzw. Sprechereinstellung).

Nach dieser Einschränkung lässt sich das Schema für propositionale Relationen auch auf die epistemische Ebene erweitern:

\section{Schema für die epistemische Lesart: R(Antez, EPS(KonsEQ))}

Die metaphorische Verwendung des Konsequens kann laut Sweetser durchaus ohne besondere lexikalische Markierung erfolgen, allerdings ist es auch möglich, dass die Sprechereinstellung im externen Konnekt overt durch einen epistemischen Operator wie ich glaube, möglicherweise, hoffentlich usw. markiert ist. Hier folgen wir den Festlegungen in HDK-1 und ,nennen die durch die betreffenden Adverbien ausgedrückten Einstellungen ,epistemisch', obwohl sich z.B. die Bedeutung von hoffentlich und leider nicht auf [i.e.S.] epistemische - Erkenntnis- - Phänomene beziehen lässt“ (HDK-1: 166). Anders als in HDK-1 gehen wir aber nicht davon aus, dass die Existenz epistemischer Operatoren im KonsEQuens notwendigerweise zur Zuordnung der Relationen zur epistemischen Ebene führt. Dies wird in 4.4.3.1 genauer diskutiert.

Ein prototypischer Fall für den Bezug auf die epistemische Ebene ist der sogenannte reduktive Schluss (vgl. GDS: 2296, HDK-1: 395), siehe auch (88).

Er ist zu Hause, weil die Lichter brennen.

EPS

(90) Wenn die Lichter brennen, ist er zu Hause.

EPS

Die Tatsache, dass die Lichter brennen, verursacht nicht, dass jemand zu Hause ist. Im Gegenteil ist die Tatsache, dass jemand zu Hause ist, der Grund dafür, dass die Lichter brennen. Wie schon die klassische Aussagenlogik zeigt, ist der Rückschluss von einer wahren Konklusion auf eine wahre Prämisse nicht zwingend, im Alltag werden reduktive Schlüsse aber häufig verwendet. Ein solcher Schluss impliziert nicht, dass das Antezedens und das Konsequens (mit dieser Rollenverteilung) in einem Ursache-Wirkung-Verhältnis stehen. Stattdessen wird in (89) und (90) von dem Antezedens auf das Konsequens geschlossen.

\subsubsection{Die Sprechaktebene}

Die Sprechaktebene unterscheidet sich funktional von der epistemischen Ebene nur dadurch, dass in diesem Fall das Konsequens keine epistemisch bewertete Proposition, sondern ein Sprechakt ist. Die Proposition des Antezedens-Konnekts bezieht sich auf diesen Sprechakt: 
(91) Was machst du heute Abend? Denn ich möchte etwas unternehmen.

(92) Zieh dir die Mütze an, obwohl ... es ist doch nicht so kalt draußen.

Auch im Falle der Sprechaktebene gilt, dass nicht nur Fragen, sondern auch andere Typen von Sprechakten wie Befehle, Bitten usw. in diese Kategorie gehören.

(91a) KAUS (AnTEz, FRAGE(Konseq))

(92a) KONZ (Antez, BITTE(KonsEQ))

Deswegen wird der allgemeine Sprechaktoperator ILL (Illokution, Sprechakt) eingeführt, mit dessen Hilfe ein analoges Schema zur epistemischen Ebene vorgeschlagen wird.

Wie im Falle epistemischer Relationen kann der Sprechakt, auf den sich die AnTezeDENS-Proposition bezieht, entweder overt durch typische Sprechaktmarker wie einen bestimmten Satzmodus, steigende oder fallende Intonation usw. markiert sein oder covert bleiben. Auf der Sprechaktebene wird zwischen diesen zwei Fällen funktional nicht unterschieden. Das Konsequens-Relat muss so oder so ein Sprechakt sein, wenn die Relation eine sprechaktbezogene Lesart haben soll. Dieser muss als solcher nicht zwingend lexikalisch markiert sein. Umgekehrt gibt es Situationen, in denen die Präsenz von overten Sprechaktmarkern noch nicht ausreicht für die Zuordnung zur Sprechaktebene, weil sich deren Skopus auf die gesamte Relation ausdehnt, wie dies in 4.4.3.1 diskutiert wird.

\subsubsection{Korrelationen zwischen den formalen Eigenschaften der Relation und ihrer Interpretation}

Ob eine Antezedens-Proposition sich auf die Proposition des Konsequens, die Sprechereinstellung zu dieser Proposition oder auf einen im Konsequens-Konnekt ausgedrückten Sprechakt bezieht, kann nicht immer ohne einen breiteren Kontext und situatives Wissen ganz eindeutig bestimmt werden. Relationen können hinsichtlich der Ebenen auch ambig bleiben, solange dies zu keinem Kommunikationsproblem führt. In vielen Fällen macht es keinen wesentlichen Unterschied in der Kommunikation, ob der Sprecher beispielsweise eine Annahme begründet oder die entsprechende Aussage (vgl. Knott/Sanders 1998 für einen analytischen und Breindl/Walter 2009 für einen korpuslinguistischen Nachweis der These der mehrfachen Lesart-Zuordnung). Will ein Sprecher seine im KonsEquens-Konnekt ausgedrückte Äußerung eindeutig als Sprechereinstellung oder Sprechakt vermitteln, stehen ihm sprachliche Mittel zur 
Verfügung wie spezifische Verbstellung im Antezedens-Konnekt, ein bestimmter Grad der syntaktischen und prosodischen Integration des Antezedens-Konnekts in sein Bezugskonnekt oder spezifische lexikalische Indikatoren der Bezugsebenen. Sie treten meistens in einem Bündel auf, sind zum Teil skalierbar und können in Kombination oder auch einzeln die Lesart unterschiedlich stark steuern. In der Literatur ist eine Reihe von Bedingungen, die die eine oder andere Lesart ausschließen, bereits hinreichend dokumentiert (vgl. Sweetser 1990: 76ff., Günthner 1993, GDS, HDK-1: 331-334, Breindl/Waßner 2006: 53f., Blühdorn 2008, Breindl/Walter 2009, Volodina 2011a). Dies betrifft konditional basierte Subjunktorrelationen, die systematisch auf allen drei Ebenen verknüpfen können. Wie einige korpuslinguistische Studien der letzten Jahre belegt haben, ist es außerdem nicht sinnvoll,

a) für semantisch unterschiedlich kodierte Relationen gleiche Korrelationen von Form und Verknüpfungstyp anzunehmen (vgl. Volodina 2011a, 2011b);

b) zu unterstellen, dass Form-Funktion-Korrelationen bei subordinativen Verknüpfungen die gleiche Natur wie die bei den parataktischen oder den koordinativen Verknüpfungen hätten. Ein postulierter Ebenenunterschied zwischen weil und denn oder zwischen weil-VL und weil-V2 korreliert mit ganz anderen Unterschieden in der syntaktischen und prosodischen Struktur als ein - ebenfalls in der Literatur oft postulierter - Ebenenunterschied zwischen deshalb und nämlich;

c) von einer eins-zu-eins-Beziehung zwischen einem bestimmten Konnektor und einer gewissen Bezugsebene, sprich von der lexikalischen Spezifik eines Konnektors auszugehen (vgl. Breindl/Walter 2009);

d) für beliebige semantische Relationen anzunehmen, dass sie genauso wie die kausalen nach den drei Ebenen der Verknüpfung differenziert sind (vgl. z.B. die Skepsis einer Übertragung des Modells auf adversative Relationen in Lang 2000).

Es hat sich gezeigt, dass die folgenden Merkmale, die einzeln oder auch mit den anderen zusammen auftreten können, durchaus als tendenzielle Hinweise auf das Vorliegen oder den Ausschluss einer bestimmten Lesart dienen können:

(i) Vorkommen von skopusfähigen Ausdrücken bzw. die Möglichkeit der Einfügung eines Korrelats im KonSEQUENS-Konnekt;

(ii) syntaktische und prosodische Integration/bzw. Desintegration des AntezeDENS-Konnekts;

(iii) Erfragbarkeit des AntezedEns-Konnekts.

Die oben genannten Merkmale werden in diesem Kapitel exemplarisch diskutiert. Für die ausführliche Beschreibung der einzelnen Relationen bezüglich des Merkmals „Ebene der Verknüpfung“ sei hier auf die entsprechenden Abschnitte in den jeweiligen C-Kapiteln verwiesen. 


\subsubsection{Auftreten von skopusfähigen Ausdrücken (Negation, Korrelate, Fokuspartikeln) im KonSEQUENS-Konnekt}

Steht das interne Konnekt im Skopus der Negation des externen Konnekts, was eine mögliche Lesart in (93a) und die einzig mögliche in (93b) ist, wird die Relation ausschließlich propositional gelesen. Nimmt die Negation engen Skopus wie in (94a), wird dagegen neben der propositionalen auch die epistemische Lesart zugänglich, wie in (94b):

(93a) Er hat sie nicht getötet/ weil sie geSCHRIen hat. $\quad \neg$ (p, weil q)

(93b) Er hat sie nicht [deshalb] getötet/ weil sie geSCHRIen hat, [sondern weil sie ihm untreu war]. PRO

(94a) Er hat sie NICHT getötet \weil sie geSCHRIen hat. $\quad(\neg p)$ weil q

(94b) Er hat sie NICHT getötet \weil sie geSCHRIen hat. [d.h. sie war noch am Leben, sonst hätte sie nicht schreien können] \#PRO

(94c) Er hat sie NICHT getötet\ weil sie geSCHRIen hat

[wenn sie nicht geschrien hätte, hätte er sie umgebracht]. PRO

Wenn also die Negation weiten Skopus über die gesamte Relation nimmt, dann liegt eindeutig eine propositionale Relation vor, wenn aber die Negation engen Skopus nimmt, sind sowohl propositionale als auch nicht-propositionale Lesarten möglich. In (94c) liegt eine Lesart mit engem Skopus vor, die besagt, dass der vom Sprecher gehörte Schrei der Frau ein Indiz dafür ist, dass sie noch am Leben sein kann. Während (94a) hinsichtlich der Verknüpfungsart ambig ist und je nach Skopuslesart unterschiedliche Interpretationen erlaubt, kann (93) mit weitem Skopus nur propositional gelesen werden.

Die Möglichkeit der Einfügung eines Korrelats, das als Kopf einer attributiven Subjunktorphrase fungiert (vgl. HDK-1: 248ff.), dient als Test für die Abgrenzung der Relationen mit der propositionalen Lesart von den anderen. Er ist allerdings nur bei Relationen anwendbar, die generell attributive Korrelatkonstruktionen zulassen, wie z.B. die Subjunktorphrasen mit weil:

Peter hat Angst, weil seine Hände zittern.

PRO/EPS

(96) Peter hat DESwegen Angst, weil seine Hände zittern. (Möglicherweise kriegt er auch die Parkinson-Krankheit wie sein Opa) PRO

Peter muss (^deswegen) Angst haben, weil seine Hände zittern. EPS

Korrelatkonstruktionen sind insbesondere für die kausalen Relationen typisch, da diese überwiegend antiikonisch, also mit nachgestelltem AnTEzedEns verwendet werden (vgl. die Liste der Kombinationsmöglichkeiten in HDK-1: 261-263): 
(98) Von Globalisierung sollte dabei jedoch schon deshalb nicht gesprochen werden, weil sich die Ausweitung der Waren- und Finanzströme weitgehend auf den Bereich der OECD-Länder beschränkt. (Frankfurter Rundschau, 12.02.1998, S. 8) PRO

Nicht untypisch ist das Eintreten einer Korrelatkonstruktion oder die Möglichkeit, ein Korrelat einzufügen, auch im Falle einer temporalen oder konditionalen Relation, wo es ebenfalls als ein Indiz für die propositionale Lesart der Relation gilt (vgl. HDK-1: 262; zu nicht-propositionalen Interpretationen von bestimmten Korrelatkonstruktionen hingegen vgl. Breindl/Walter 2009: 141):

(99) Was der Großstädter nicht weiß: Im Ökosystem des Waldes mit seinen vielfältigen Abläufen enden die Anpflanzungen immer dann, ehe die Bäume beginnen, ihre Blüten zu treiben. (Süddeutsche Zeitung, 26.05.1998, S. 41)

(100) In Großbritannien erhält man Arbeitslosengeld höchstens 312 Tage lang und nur dann, wenn man normalerweise als Arbeitnehmer tätig ist. (Die Presse, 14.02.1996, o.S.)

PRO

Wie oben bereits erwähnt, kommen nicht alle Konnektoren und alle Relationen in solchen Korrelatkonstruktionen vor. Abgesehen von den Adverbkonnektoren wie folglich, schließlich, also sowie dem syntaktischen Einzelgänger denn, die aufgrund ihrer syntaktischen Eigenschaften nicht skopusfähig sind, lassen auch die Postponierer wie weshalb, weswegen und sodass keine Korrelate zu. Es wäre aber falsch anzunehmen, dass die mit den genannten Postponierern eingeleiteten Relationen deswegen nicht propositional gelesen werden können.

Ebenfalls liegt die propositionale Lesart einer Relation nahe, wenn das interne Konnekt durch Fokuspartikeln wie nur im externen Konnekt fokussiert wird:

(101) Ein Erfolg ist nur beschieden, wenn alle Massnahmen als Ganzes greifen können. (St. Galler Tagblatt, 30.04.1997, o.S.) PRO

Als Träger der epistemischen Bedeutung können Adverbien und matrixsatzähnliche Ausdrücke der epistemischen Einstellung die epistemische Lesart der Relation dennoch nicht immer garantieren. Das liegt unter anderem daran, dass epistemische Operatoren wie ich glaube, wenn sie satzinitial vorkommen, sowohl weiten wie in (102a) als auch engen Skopus wie in (102b) nehmen können, wobei die epistemische Lesart nur dann garantiert ist, wenn sie mit engem Skopus auftreten.

(102a) Ich glaube, dass Peter krank ist $\backslash$ weil er beim ARZT war (und sich im Wartezimmer angesteckt hat).

\#EPS 
(102b) Ich glaube, dass Peter KRANK ist/ weil er beim ARZT war. (Nämlich immer wenn Peter krank ist, geht er zum Arzt)

EPS

Epistemische Adverbien wie vielleicht, vermutlich, wahrscheinlich, die eine bestimmte Einstellung des Sprechers hinsichtlich der Wahrscheinlichkeit der Geltung des im KonSEquENS-Konnekt ausgedrückten Sachverhalts ausdrücken, oder solche wie leider, hoffentlich, erfreulicherweise, die auf die emotionale Einstellung des Sprechers zum KonSEQuens-Sachverhalt verweisen, verhalten sich anders. Im Unterschied zu matrixsatzähnlichen Ausdrücken wie ich glaube oder ich hoffe, die ebenfalls auf der epistemischen Ebene fungieren können, können Adverbien nur engen Skopus haben, wenn sie ohne einen eindeutigen Skopusmarker (wie z.B. ein pronominales Korrelat wie in 102d) vorkommen:

(102c) Peter ist vielleicht KRANK\ weil er beim ARZT war. (Immer wenn Peter krank ist, geht er zum Arzt)

EPS

(102d) Peter ist vielleicht deswegen krank/ weil er beim ARZT war. (Er hätte sich im Wartezimmer anstecken können.)

\#EPS

(102e) ?Vielleicht ist Peter krank/ weil er sich bei Paul angesteckt hat. EPS/PRO

Dies zeigt, dass die Präsenz epistemischer Adverbien sowie von Adverbien der Sprechereinstellung weitestgehend als ein hinreichendes Indiz für die epistemische Lesart der Relation angesehen werden kann, während Relationen mit matrixsatzähnlichen epistemischen Operatoren zwar eine epistemische Lesart haben können, diese jedoch für sie nicht zwingend ist. (Zum Unterschied zwischen Satzadverbien und semantisch weitgehend gleichen matrixähnlichen Ausdrücken s. Lang 1979, ferner Volodina 2011a).

Bei Sprechaktoperatoren wie ich behaupte, ich befehle dir, ich frage dich, die den vollzogenen Sprechakt selbst benennen, wurden dieselben Skopusambiguitäten wie bei epistemischen Operatoren festgestellt: Sie können, wenn sie mit engem Skopus auftreten, dazu beitragen, dass die Äußerung auf der Sprechaktebene zu lesen ist.

(103) Ich behaupte deswegen, dass Peter krank ist, weil er nicht zur Schule kam. (Warum behauptest du, dass Peter krank ist?) SPA

(104) Ich behaupte, dass Peter deswegen krank ist, weil er nicht zur Schule kam. (Warum ist Peter krank?) \#SPA

Während die Präsenz performativer Sprechaktoperatoren allein nicht die Lesart der Relation auf der Sprechaktebene bewirken kann, ist das Vorkommen des Sprechaktoperators bitte im KonSEQUENs-Konnekt hinreichend für die sprechaktbezogene Lesart der Relation. Bitte verhält sich auf der Sprechaktebene ähnlich wie die epistemischen 
Adverbien, nimmt also ausschließlich engen Skopus und fungiert daher als typischer Sprechaktoperator.

(105) Würdest du bitte das Radio ausmachen\ weil ich morgen eine Klausur schreibe.

SPA

(106) Wenn du nach Hause kommst \ruf mich bitte an!

SPA

In Bezug auf das Merkmal „Auftreten von skopusfähigen Ausdrücken im KonsEQuensKonnekt“ lässt sich festhalten, dass die Präsenz solcher Ausdrücke tendenziell eine ebenendisambiguierende Rolle hat. Epistemische Satzadverbien und matrixsatzähnliche Operatoren sowie Sprechaktoperatoren, die Ausdrücke als Gedanken bzw. Sprechakte explizit markieren, können genau dann als Marker der epistemischen oder Sprechaktebene angesehen werden, wenn sie engen Skopus haben.

\subsubsection{Syntaktische Einbettung des Antezedens-Konnekts in die Struktur seines Bezugskonnekts}

Das Antezedens-Konnekt kann in die Struktur seines Bezugskonnekts integriert oder auch nichtintegriert vorkommen. Der höchste Grad syntaktischer und prosodischer Integration (im Sinne des in HDK-1 und in A1.2 dargelegten Konzepts der syntaktischen Einbettung) des Antezedens-Konnekts liegt vor, wenn es im Vorfeld einer Konstruktion und zusammen mit seinem Bezugskonnekt als eine einzige prosodische Einheit realisiert wird, d.h. das erste Konnekt mit steigender Tonhöhenbewegung (/) endet, womit dem Hörer signalisiert wird, dass die Äußerung noch nicht abgeschlossen ist, wie in (107a) gezeigt. Wir sprechen von der Desintegration einer Subjunktorphrase, wenn die Konnekte zwar semantisch eine Einheit bilden, das AntezedensKonnekt dagegen syntaktisch nicht in die Struktur des KonSEquens-Konnekts eingebettet ist, wobei am Ende des ersten Konnekts ein separierender Grenzton (\) festzustellen ist, wie in (107b) und (108). Hinsichtlich des Zusammenhangs zwischen der Ausprägung des Grades der Integration des Antezedens-Konnekts und der Lesart der Relation besteht in der Literatur weitgehend Einigkeit: Die syntaktische und/oder prosodische Desintegration einer Subjunktorphrase begünstigt nicht-propositionale Lesarten, wogegen hohe syntaktische und prosodische Integration des AntezedensKonnekts eine propositionale Lesart nahe legen (vgl. Pasch 1997, Günthner 2000, Scheutz 1998b, Blühdorn 2008, Breindl 2008a, Antomo/Steinbach 2010, 2013):

(107a) Wenn du mich fragst/ machte ich das für dich. PRO

(107b) Wenn du mich fragst $\backslash$ ich würde mit dem Typ nie ausgehen. \#PRO

(108) Ich finde ihn nett $\backslash$ obwohl ... irgendwas stört mich bei ihm doch. 
Da diese Generalisierung eine binäre Opposition von Ebenen zugrundelegt, ist sie viel zu allgemein für ein Drei-Ebenen-Modell, deswegen muss sie hier präzisiert werden: Sie trifft zu, wenn es z.B. darum geht, zwischen den propositionalen und epistemischen Lesarten einer kausalen Relation - KAUS(Antez, Konseq) vs. KAUS (Antez, EPS(Konseq)) - zu unterscheiden, zwischen den propositionalen und spechaktbezogenen Lesarten einer kausalen Relation - KAUS(Antez, Konseq)) vs. KAUS(ANTEZ, ILL(KonSEQ)) - oder zwischen den propositionalen und spechaktbezogenen Lesarten einer konditionalen Relation zu unterscheiden - KOND(ANTEz, KonSEQ) vs. KOND(ANTEz, ILL(KonSeQ)) -, aber nicht zwischen den propositionalen und epistemischen Lesarten einer konditionalen Relation - KOND(AnTEz, Konseq) vs. KOND(Antez, EPS(KonSEQ)) -, denn die propositionalen und epistemischen Konditionalen weisen gleiche syntaktisch-prosodische Kodierungsmuster auf (vgl. C4.1.2.4, Abb. C 4.1-3).

(109) Wenn sie geschieden ist/ war sie verheiratet. (Bsp. nach Sweetser 1990: 114)

EPS

(110) Wenn es schneit/ ist der Besitzer des Grundstückes dazu verpflichtet, den Schnee zu räumen und gegen Glatteis zu streuen. PRO

Vielfach wird in der Literatur darauf hingewiesen, dass die Nichtvorfeldfähigkeit eines weil-Satzes seine propositionale Lesart blockiert. Dieser Test filtert zwar die epistemischen weil-V2-Sätze wie in (111a) aus, deren AnTEZEDENs-Konnekt syntaktisch nicht eingebettet werden kann (vgl. 111b), und die epistemischen weil-VL-Sätze, denen ein reduktiver Schluss zugrunde liegt (vgl. 111c), nicht aber die weil-VL-Sätze, deren KonSEquens-Konnekt ein epistemisches Modalverb enthält, wie (111d):

(111a) Es hat geregnet \weil die Straße ist nass.

EPS

(111b) *Weil die Straße ist nass/ hat es geregnet.

(111c) \#Weil die Straße nass ist/ hat es geregnet. \#EPS

(111d) Weil die Straße nass ist/ muss es geregnet haben. EPS

Ähnlich wie weil-V2-Strukturen verhalten sich auch u.a. adversative während- und konzessive obgleich-, obschon-, obwohl- und wobei-Sätze, die statt der kanonischen Verbletztstellung in bestimmten Kontexten auch V2-Stellung des Finitums aufweisen können (zur Problematik nicht-kanonischer V2-Stellung in adverbialen Nebensätzen, nicht-restriktiven Relativsätzen und dass-Sätzen siehe Antomo/Steinbach 2010, 2013, Breindl 2009, Gärtner 2001, Holler 2008, Freywald 2008, Reis 2013).

(112) Aber des ist doch im wesentlichen des, woraus die Stadt Worms ihren Etat finanziert. Während die große Masse der sonst aufzuwendenden Steuern gehen ja entweder nach Bonn, an die Bundesregierung oder an die Lander, nach Mainz.

(Pfeffer-Korpus, Deutsche Umgangssprache, PF 100) 
(113) Österreichs Bundeskanzler Vranitzky ist auch Politiker, obschon: Körperhaltung und Diktion lassen ja mitunter vermuten, das er das ungern ist. (Die Presse, 12.01.1996, S. 1)

(Beispiele aus Breindl 2009: 284)

Ein V2-Satz als Antezedens-Konnekt ist in die Struktur seines Bezugskonnekts nicht integriert, daher sind die V2-Strukturen generell semantisch und pragmatisch flexibler und aus diesem Grund werden sie präferiert auf einer der nicht-propositionalen Ebenen interpretiert (mehr dazu in C4.2.2.4, C4.3.4):

(114) Ausländer-Vignette nicht „wasserfest“: Nur ein einziger Wassertropfen genügt, um den per Tintenstrahl aufgedruckten EU-konformen „Stempel“ auf dem Pickerl zu verwischen - und so wird man künftig Gendarmen und Polizisten immer öfter sagen hören: „Ausweiskontrolle, kommen s in die Hauseinfahrt, weil dort regnet es nicht.“ (Neue Kronen-Zeitung, 27.03.1999, S. 16)

Die V2-Stellung im AnTEZEDEns-Konnekt ist jedoch nicht hinreichend, um gleich jeden V2-Nebensatz als epistemisch einzustufen, vgl. (115) am Beispiel von weil. Umgekehrt können auch nachgestellte weil-VL-Sätze epistemische Lesarten haben, insbesondere dann, wenn das weil-Konnekt prosodisch desintegriert vorkommt (vgl. 116b):

(115) Ich hab' die Mauer auch gesehen, am Brandenburger Tor. Ich am Alexanderplatz. Und früher konnte man da nicht rüber, weil da war keine Leiter und keine Treppe. Da durfte man auch gar nicht rüber. PRO

(Beispiel aus Breindl/Walter 2009: 161)

(116a) Peter ist KRANK\weil er war beim ARZT. (Immer wenn Peter krank ist, geht er zum Arzt.)

EPS

(116b) Peter ist KRANK \ weil er beim ARZT war. (Immer wenn Peter krank ist, geht er zum Arzt.)

EPS

Darüber hinaus wirkt nicht nur fallende Tonhöhenbewegung an der syntaktischen Grenze zwischen den Konnekten als desintegrierend, sondern auch stark steigende, unter der Bedingung, dass der Neuansatz wesentlich tiefer ansetzt als die letzte Silbe der ersten Intonationseinheit. Wird das KonsEquens-Konnekt wie in (116c) mit stark steigender Tonhöhenbewegung realisiert, liegt eine Frage vor, die im AntezedensKonnekt begründet wird. Dies macht die sprechaktbezogene Lesart möglich.

(116c) Peter ist KRANK? Weil er war beim ARZT.

SPA

Präferiert sprechaktbezogen werden kausale, konzessive und konditionale Relationen gelesen, wenn das Antezedens-Konnekt an der Nullstelle (Vor-Vorfeld), also syntaktisch und prosodisch desintegriert, vorkommt: 
(117) Weil du das Handy dabei hast: Könntest du bitte die Polizei alarmieren?

(118) Obwohl du mich kein einziges Mal angerufen hast: Hier sind zwei Kinokarten für heute.

(119) Auch wenn das nun alles etwas negativ klingt: Journalistin zu sein ist für mich nach wie vor einer der spannendsten Berufe, den ich kenne.

(http://www.ucip.ch/th/kg)

V1-Konditionalsätze mit Sprechaktbezug sind ausgeschlossen, nicht aber mit Subjunktoren eingeleitete Konditionalsätze:

(120a) Wenn wir gerade dabei sind: Wo bist du denn gestern gewesen?

(120b) * *ind wir grade dabei: Wo bist du denn gestern gewesen?

Temporale, irrelevanzkonditionale und kontrafaktische konditionale Relationen sind bezüglich des Faktors „Desintegration des Antezedens-Konnekts“ unterspezifiziert: Die Irrelevanzkonditionalen haben eine generelle Tendenz zur Desintegration des anteponierten Antezedens-Konnekts (vgl. König/Van der Auwera 1988, Pittner 1999, d'Avis 2004, siehe C4.4), vgl. (121)).

(121) Ob wir hier rauskommen oder nicht, die Sache ist zu Ende. Finito! (DIV/SHT, Schädlich, Trivialroman, S. 70)

\#PRO

Kontrafaktische wenn-Sätze sind unabhängig von dem Unterschied in der syntaktischen Struktur in zwei bedeutungsgleichen Varianten belegt, siehe (122) (s. C4.1.2.4.1):

(122a) Wenn ich ein ordentlicher Hund wäre, ich täte es nicht fressen.

(Bsp. nach Volodina 2011a: 140)

(122b) Wenn ich ein ordentlicher Hund wäre, täte ich es nicht fressen.

Zwar lässt sich an vielen Beispielen zeigen, dass sich in Bezug auf das Merkmal „Syntaktische Einbettung des Antezedens-Konnekts“ die epistemische Ebene und die Sprechaktebene (je nach der Art der Relation) in vielen Hinsichten restriktiver verhalten als die propositionale Ebene. Formale Merkmale und die ebenenbezogene Lesart stehen aber nicht immer in einem eins-zu-eins-Verhältnis zueinander.

\subsubsection{Erfragbarkeit des Antezedens-Konnekts}

Ein weiteres Merkmal für die Unterscheidung zwischen den ebenenbezogenen Lesarten ist die Erfragbarkeit des Antezedens-Konnekts, d.h. je nach Lesart ist die Realisierung einer Relation in Frage-Antwort-Paaren, wobei in der Antwort-Sequenz 
das KonSequens unter Ellipse des Bezugssatzes (vgl. Reis 1997) vorkommt, zugelassen oder nicht. Dieses Merkmal trifft jedoch nicht auf alle Relationen zu, da es zahlreiche Relationen gibt, die nicht erfragbar sind, und zwar in erster Linie Relationen, die als parataktische Strukturen realisiert werden.

Die Möglichkeit, das Antezedens-Konnekt als elliptische Antwort auf eine entsprechende $W$-Frage zu äußern, wobei das externe Konnekt aus der Frage zu rekonstruieren ist, ist ein Indiz für die propositionale Lesart einer konditional basierten Subjunktorrelation:

(123) Peter ist nicht am Institut, weil er Urlaub hat. PRO Warum ist Peter nicht am Institut? Weil er Urlaub hat.

(124) Peter hat Urlaub, weil er nicht am Institut ist. EPS *Warum hat Peter Urlaub? Weil er nicht am Institut ist.

(125) Wenn Peter im Urlaub ist, ist er nicht am Institut. PRO In welchem Fall ist Peter nicht am Institut? Wenn er im Urlaub ist.

(126) Wenn Peter nicht am Institut ist, hat er Urlaub. EPS *Unter welchen Umständen hat Peter Urlaub? Wenn Peter am Institut ist.

Hierbei ist allerdings wichtig zu bemerken, dass die $W$-Frage ohne eine epistemische oder Sprechaktmodifikation des Konsequens-Konnekts erfolgen muss, d.h. die $W$ Frage in (124) kann nicht als Test für die propositionale Ebene angesehen werden. Eine weitere Besonderheit ist, dass bei kausalen Relationen die propositionale Lesart durch Warum-Fragen angezeigt wird, da im Falle epistemischer und sprechaktbezogener Lesart Wieso-Fragen akzeptabler sind (vgl. Keller 1993, Günthner 1993).

(124a) Peter hat Urlaub, weil er nicht am Institut ist. \#PRO \#Warum/Wieso hat Peter Urlaub?

Weil er nicht am Institut ist.

Festzuhalten ist noch, dass dieser Test nicht immer anwendbar ist, da in der Regel aus dem Kontext rekonstruiert werden muss, ob das externe Konnekt eine geeignete Antwort auf eine entsprechende Frage wäre. 


\subsubsection{Fazit und Anmerkungen zur weiteren Vorgehensweise im Handbuch}

Das Ziel dieses Kapitels war, den Beitrag darzustellen, den Sweetsers Drei-EbenenTheorie zur Beschreibung und Analyse solcher konditional-basierten und temporalen Subjunktorrelationen liefert, in denen Sprechakte und epistemische Einstellungen als Relata fungieren. Hierbei standen die Begriffsklärung und die Diskussion der theoretischen Relevanz der einzelnen Bezugsebenen im Vordergrund. Es wurden drei empirisch testbare Merkmale vorgestellt, die auf den Merkmalen Skopuseigenschaften von Operatoren im KonSEquEnS-Konnekt, syntaktische Einbettung und prosodische Integration des Antezedens-Konnekts sowie Erfragbarkeit des AntezedensKonnekts basieren. Diese Tests tragen bündelweise zur Zuordnung von Äußerungen zu den einzelnen Bezugsebenen bei bzw. helfen, eine der Lesarten auszuschließen. Die Unterschiede zwischen den Bezugsebenen können wie folgt festgehalten werden:

Strukturen, die auf der propositionalen Ebene fungieren,

a) können im Skopus der Negation und skopusfähiger Ausdrücke wie Fokuspartikeln stehen;

b) können als attributive Korrelatstrukturen realisiert werden;

c) sind erfragbar, d.h. ihr internes Konnekt kann eine $W$-Frage direkt beantworten;

d) sind prosodisch in der Regel als eine einzige Intonationseinheit realisiert.

Strukturen, die auf der epistemischen Ebene fungieren,

a) sind häufig durch syntaktische und prosodische Desintegration des internen Konnekts gekennzeichnet (nicht notwendig für die konditionale Relation);

b) können nicht im Skopus der Negation und skopusfähiger Ausdrücke wie Fokuspartikeln wie nur stehen;

c) können in der Regel nicht als Korrelatstrukturen realisiert werden;

d) sind nicht direkt erfragbar, d.h. sie können nicht im Skopus einer $W$-Frage stehen;

e) sind durch die Präsenz von epistemischen Adverbien gekennzeichnet, die engen Skopus nehmen.

Strukturen, die auf der Sprechaktebene fungieren,

a) sind häufig durch syntaktische und prosodische Desintegration des internen Konnekts gekennzeichnet;

b) können in der Regel nicht als Korrelatstrukturen realisiert werden;

c) sind nicht direkt erfragbar, d.h. sie können nicht im Skopus einer $W$-Frage stehen; 
d) sind durch die Präsenz von Sprechaktoperatoren gekennzeichnet, die jedoch nur dann die sprechaktbezogene Lesart begünstigen, wenn sie engen Skopus nehmen.

Die Unterspezifikation der Form-Lesart-Korrelation, die durch die Anwendung der genannten Tests deutlich wird, begründet die Notwendigkeit einer detaillierteren Darstellung in den jeweiligen C-Kapiteln zu den einzelnen Relationen. 